\title{
SYMMETRICAL OBSERVABILITY OF KINEMATIC PARAMETERS IN SYMMETRICAL-PARALLEL MECHANISMS
}

\author{
S. Durango ${ }^{1, \dagger}$, D. Restrepo ${ }^{2}$, O. Ruiz ${ }^{2}$, J. Restrepo-Giraldo ${ }^{2}$, S. Achiche ${ }^{3}$ \\ ${ }^{1}$ Grupo de investigación DM-DI, Universidad Autónoma de Manizales \\ † Corresponding author (sebastiandi@autonoma.edu.co) \\ ${ }^{2}$ Laboratorio de CAD CAM CAE, Universidad EAFIT \\ ${ }^{3}$ École Polytechnique de Montréal, Université de Montréal
}

\begin{abstract}
This article presents an application of symmetry groups in symmetrical observability of kinematic parameters of parallel mechanisms. The devised concepts are used in the domain of Kinematic Identification (KI). The investigation takes advantage of the mechanism structural symmetries and presents the following contributions: (i) a conjecture that allows mapping the symmetries of the mechanism into the articular-variables space, (ii) the necessary conditions to express leg parameters in coordinate systems which allow symmetrical observability, and (iii) a procedure for exploiting symmetries in pose selection for KI that reduces the design-of-experiments costs to $\left(1 / n_{\text {legs }}\right)$ when compared to a KI procedure in which each leg configurations are selected independently. An application of the symmetrical observability is presented through the simulated KI of a $3 \underline{R} R R$ symmetrical parallel mechanism.
\end{abstract}

Keywords: Symmetrical observability, kinematic identification, parallel mechanism.

\section{INTRODUCTION}

Reference [7] defines a fully-parallel mechanism as a closed-loop mechanism in which an $n$ Degrees-of-Freedom (DOF) mobile platform is connected to a fixed base by $n$ independent kinematic chains (legs), each having a unique actuated joint. Most of fully-parallel mechanisms are formed by a symmetrical structure. A symmetrical-parallel mechanism is defined as a fully-parallel one with two additional conditions $[12,13]$ :

1. Each leg is formed by an identical kinematic chain.

2. In at least one particular configuration the kinematic structure defines a symmetry group $G_{M}$. 
The analysis of the symmetrical characteristics of parallel mechanisms is one of the least-studied problems. Literature is restricted to workspace and singularity analyses. Reference [12] presents a symmetric theorem of workspace for symmetrical-parallel mechanisms. The theorem reveals an analogous relationship between the workspace shape and the symmetrical structure. This theorem is proposed to estimate geometrical characteristics of the workspace and to guide the conceptual design of spatial parallel manipulators. The theorem is limited to mechanisms in which each identical kinematic chain (leg) always remains collinear. In [13] the symmetrical workspace theorem is strengthened to include a general category of symmetrical parallel mechanisms in which the permanent collinearity of the legs is not required. Reference [14] presents an application of the symmetrical workspace theorem that addresses the symmetrical calculation of singularities of symmetrical-parallel mechanisms. A common characteristic of $[13,14]$ is the use of symmetry groups theory for proving the symmetrical theorems. Different from [12, 13, 14], reference [2] presents a methodology based in a parametric representation of the orientation for the workspace and singularity symmetrical analyses of spherical parallel mechanisms. Finally, reference [3] sketches the use of symmetries in the kinematic calibration of a Gough platform.

This article extends the use of structural symmetries in parallel mechanisms addressing the problem of formulating symmetrically observable sets of leg parameters for Kinematic Identification (KI). The main condition for symmetrical observability is the workspace symmetry that was probed for symmetrical-parallel mechanisms in $[12,13]$. If a linear model (joint gain and offset) is assumed for the articular coordinates, then the articular-variables space symmetry is required too. The proof of an articular-variables symmetrical space theorem is analogous to the forward kinematics problem of parallel mechanisms that in general has only numerical solution [8]. In consequence, a conjecture for the articular-variables space symmetry is proposed in section 3 .

A natural use for the symmetrical observability would be an inverse KI in which the identification experiments are planed for a reference leg only and extended to the remaining $n-1$ legs by symmetrical operations. The KI protocol by [5] was updated with a symmetrical pose selection procedure (see section 4.1). The updated procedure has an advantage with respect to [5]: the cost of designing identification experiments is reduced to $1 / n_{\text {legs }}$ by the use of observability symmetries.

The layout for the rest of the article is in the following manner: Section 2 presents fundamentals of symmetry groups theory. A theorem of symmetrical workspace of symmetricalparallel mechanisms is extended on section 3 proposing a conjecture for articular-variables symmetrical space. The symmetrical observability of sets of leg parameters is proposed in section 4, and its application in KI is presented in section 4.1. Results are presented in section 5 through a $3 \underline{R} R R$ fully-parallel mechanism in which an application of symmetrical observability of sets of leg parameters is used in a simulated KI. Finally, concluding remarks are presented in section 6 .

\section{FUNDAMENTALS OF SYMMETRY GROUPS}

A group is a set $G$ equipped with an internal binary operation $\odot$ such that the operation is associative, with closure, and has a neutral and an inverse element in $G$ [9]. Two instances 
of groups are used to describe symmetries of the structure, workspace and observability of parameters in symmetrical-parallel mechanisms: The symmetry group $\Sigma$, section 2.1 , and the dihedral group $D_{2 n}$, section 2.2.

\subsection{Symmetry group $\Sigma$}

Let $V$ a polygon in the plane. The symmetry group $\Sigma(V)$ consists of all the rigid motions $\lambda$ for which $\lambda(V)=V$, that is, the symmetry group is formed by the operations that allow the polygon to superimpose with itself [13].

\subsection{Dihedral group $D_{2 n}$}

Let $V_{n}$ denotes a regular polygon with $n$ vertices and center $O$. The vertices of $V_{n}$ are denoted as $v_{i}(i=1,2, \ldots, n)$. The symmetry group $\Sigma\left(V_{n}\right)$ is called the dihedral group of $V_{n}$ with $2 n$ elements and denoted as $D_{2 n}$. The elements of the dihedral group depend on the parity of the regular polygon. A complete description of the dihedral group can be founded in [13].

As an example, consider the symmetries of the equilateral triangle shown in Fig. 1. These symmetries can be expressed by the dihedral group $D_{6}$ whose elements are permutations of the set of vertices $V=\left\{v_{1}, v_{2}, v_{3}\right\}$. In consequence, the symmetry group is defined by:

$$
D_{6}=\left\{\lambda_{1}, \lambda_{2}, \lambda_{3}, \lambda_{4}, \lambda_{5}, \lambda_{6}\right\},
$$

where $\lambda_{1}=(1)$ denotes a 0 -rotation about point $O, \lambda_{2}=(123)$ denotes a $2 \pi / 3$-rotation about point $O, \lambda_{3}=(132)$ denotes a $4 \pi / 3$-rotation about point $O, \lambda_{4}=(23)$ denotes a reflection about the line $O v_{1}, \lambda_{5}=(13)$ denotes a reflection about the line $O v_{2}$, and $\lambda_{6}=(12)$ denotes a reflection about the line $O v_{3}$, [14].

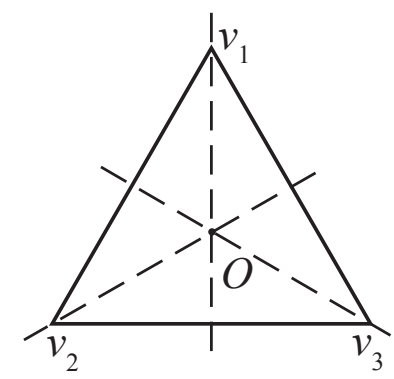

Figure 1. Reflection lines of an equilateral triangle [14].

Symmetry groups theory is used by [13] to prove the symmetrical theorem of workspace for symmetrical-parallel mechanisms. The theorem is summarized in section 3.1.

\section{ARTICULAR-VARIABLES SYMMETRICAL SPACE AND SYMMETRICAL WORKSPACE OF SYMMETRICAL-PARALLEL MECHANISMS}

Reference [10] defines the workspace of a parallel mechanism as the total volume swept out by the mobile platform as the mechanism executes all possible motions. A symmet- 
rical theorem of workspace for symmetrical-parallel mechanisms was proposed by [13] in the following manner:

\subsection{Symmetrical workspace of symmetrical-parallel mechanisms}

If the symmetry group of the workspace of a mechanism is denoted by $G_{W}$ and the symmetry group of the kinematic chain structure being the mobile platform in a particular configuration (position and orientation) is denoted by $G_{M}$, then $G_{M}$ must be a subgroup of $G_{W}$, namely, the following relation always hold

$$
G_{M} \subseteq G_{W}
$$

In consequence, if the kinematic structure of a mechanism has associated a symmetry group $G_{M}$, then the workspace $G_{W}$ remains unaltered under the symmetry operations $\lambda$ that are the elements of $G_{M}$. A proof of the symmetrical theorem of workspace is provided in [13] using symmetry groups theory.

The workspace symmetry is a necessary condition to declare symmetrically observable sets of leg parameters (see section 4). If a linear model is assumed for the articular coordinates, then an articular-variables symmetrical space is required too (see section 4). In section 3.2 the workspace symmetries are extended to the articular-variables space of symmetrical-parallel mechanisms.

\subsection{Conjecture. Articular-variables symmetrical space of symmetrical-parallel mecha- nisms}

Symmetrical-parallel mechanisms are reported by $[12,13]$ to have symmetrical structure and a correspondent symmetrical workspace. However, a symmetrical workspace is not a sufficient condition to obtain a correspondent articular-variables symmetrical space. Therefore, it is proposed a symmetrical conjecture of articular-variables space for symmetricalparallel mechanisms in the following manner:

If a symmetrical-parallel mechanism has a symmetrical workspace characterized by a symmetry group $G_{W}$, then its is possible to set a reference system for the articular variables that produces an articular-coordinates symmetrical space characterized by a symmetry group $G_{Q}$.

The conditions to configure a symmetrical-parallel mechanism with articular-variables symmetrical space are summarized in the following manner:

1. The constraint kinematic equation of each leg of the mechanism, $F_{\kappa}$, can be expressed in its implicit form:

$$
F_{\kappa}=g_{\kappa}\left(\varphi_{\kappa}, \mathbf{r}, \rho\right)-q_{\kappa}=0 \quad\left(\kappa=1,2, \cdots, n_{\text {legs }}\right),
$$

where $\kappa$ denotes the $\kappa$ th leg, $g_{\kappa}$ is an inverse kinematic function, $\varphi_{\kappa}$ is the set of kinematic parameters, $\mathbf{r}$ and $\rho$ are the generalized coordinates (position and orientation of 
the mobile platform), and $q_{\kappa}$ is the $\kappa$ th articular coordinate. The set of constraint equations for the complete mechanism are defined in the following manner:

$$
F(\mathbf{q})=\left[\begin{array}{c}
F_{1}\left(q_{1}\right) \\
F_{2}\left(q_{2}\right) \\
\vdots \\
F_{n_{\text {legs }}}\left(q_{n_{\text {legs }}}\right)
\end{array}\right]=\left[\begin{array}{c}
g_{1}\left(\varphi_{1}, \mathbf{r}, \rho\right)-q_{1} \\
g_{2}\left(\varphi_{2}, \mathbf{r}, \rho\right)-q_{2} \\
\vdots \\
g_{n_{\text {legs }}}\left(\varphi_{n_{\text {legs }}}, \mathbf{r}, \rho\right)-q_{n_{\text {legs }}}
\end{array}\right],
$$

where $\mathbf{q}=\left[\begin{array}{llll}q_{1} & q_{2} & \cdots & q_{n_{\text {legs }}}\end{array}\right]^{T}$ is the vector of articular coordinates.

2. The kinematic structure of the mechanism has associated a symmetry group $G_{M}$ in a particular configuration of the mobile platform.

3. The articular-coordinates reference system is defined such that in the particular configuration that defines the symmetry group of the mechanism structure $\left(G_{M}\right)$, the articular variables are symmetric too. The symmetry group of the articular coordinates is denoted as $G_{Q}$.

If the articular-coordinates space is symmetric, then the following relation holds:

$$
F\left(\lambda_{i}(\mathbf{q})=\lambda_{i}(F(\mathbf{q})) \quad\left(i=1,2, \cdots, n_{\text {legs }}\right),\right.
$$

where $F$ is the set of constraint kinematic equations of the mechanism (see Eq. 4) and $\lambda_{i} \in G_{Q}$ is a symmetry operation of the symmetry group of the articular-variables space.

The proof of Eq. 5 is analogous to the forward kinematics of parallel mechanisms: it requires the solution of the constraint kinematic equations given the vector of joint variables. In general it is not possible to express the forward kinematics of parallel mechanisms in an analytical manner [8]. In consequence, a proof of the symmetrical conjecture of articularvariables space is not straightforward. An analytical proof of the conjecture is not here provided. However, in section 5 the conjecture is verified through a numerical analysis of the workspace and articular-coordinates space of a three DOF parallel mechanism.

In section 4 the workspace and articular-coordinates space symmetries are used in the declaration of symmetrical sets of leg parameters with an application in KI of symmetricalparallel mechanisms.

\section{SYMMETRICAL OBSERVABILITY OF KINEMATIC PARAMETERS}

If a parallel mechanism mets the conditions of the symmetrical theorem of workspace, section 3.2 and references [12,13], then it is possible to declare its set of kinematic parameters in order to obtain a symmetrical observability of its legs. In consequence, the planning of KI experiments can be reduced according to the observability symmetry group. In order to formulate symmetrically observable sets of leg parameters the following conditions are assumed:

1. The symmetries of the mechanism structure are described by the symmetry group $G_{M}$ and the symmetrical workspace is characterized by the symmetry group $G_{W}$. The symmetrical theorem of workspace for spatial parallel mechanisms is proposed in $[12,13]$ and summarized in section 3.1 . 
2. An independent vector-loop constraint kinematic equation is written for each leg in the form of Eq. 3. The following hypothesis are assumed:

(a) Each joint (U, spherical, prismatic, revolute) is modeled as perfect.

(b) If the mechanism is planar, then all the links are modeled as constrained in the mechanism plane.

The hypothesis $(2 \mathrm{a})$ is consequent with realistic operation conditions in which the influences of joint defects have a minor effect on pose accuracy compared with errors in the location of the joints [4].

3. The position of each fixed point $A_{\kappa}$ (U-joint, spheric joint, etc.) is defined by three parameters, Fig. 2:

(a) The magnitude of the $\overline{O A_{\kappa}}$ segment $\left(\kappa=1,2, \ldots, n_{\text {legs }}\right)$.

(b) The angle $\alpha_{\kappa}$ of the $\overline{O A_{\kappa}}$ segment with respect to the $Z$ axis of the base reference system $\left(\kappa=1,2, \ldots, n_{\text {legs }}\right)$.

(c) The angle $\beta_{\kappa}$ of the projection of the segment $\overline{O A_{\kappa}}$ on plane $X Y$ with respect to the $X$ axis of the base reference system $\left(\kappa=1,2, \ldots, n_{\text {legs }}\right)$.

4. The position of each mobile point $b_{\kappa}$ (U-joint, spheric joint, etc.) is described by three parameters, defined analogously to the fixed points, Fig. 2. The ouvw reference system is analogous to the $O X Y Z$. The three parameters are denoted as $\overline{o b_{\kappa}}, \alpha_{b_{\kappa}}$ and $\beta_{b_{\kappa}}$ $\left(\kappa=1,2, \cdots, n_{\text {legs }}\right)$.

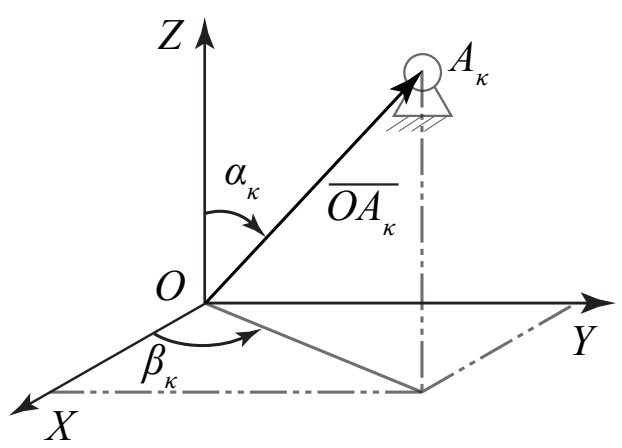

Figure 2. Position of fixed point $A_{\kappa}$.

A linear model is assumed for the articular coordinates of the mechanism in equation 6 . In consequence, two additional parameters need to be estimated for each leg: the joint gain $k_{\kappa}$, and the joint offset $\gamma_{\kappa}$. Therefore, additional symmetry conditions are required to allow a symmetrical observability:

5. Each articular coordinate has the same nominal gain $k_{\kappa}\left(\kappa=1,2, \ldots, n_{\text {legs }}\right)$.

6. The mechanism has configured an articular-variables symmetrical space described by a symmetry group $G_{Q}$. The conditions to set an articular-variables symmetrical space are proposed in section 3 , conditions (1) to (3). 
The articular coordinate linear model is defined in the following manner:

$$
\theta_{\kappa}=k_{\kappa} \psi_{\kappa}+\gamma_{\kappa} \quad\left(\kappa=1,2, \cdots, n_{\text {legs }}\right)
$$

where $\theta$ is the articular coordinate, $\psi$ is the sensor reading, $k$ is the gain in the articular variable, and $\gamma$ is the offset of the sensor.

The symmetrical observability implies that the observability of the $i$ th kinematic parameter of the $\kappa$ th leg in the $j$ th configuration must be the same that the observability of the correspondent parameter of a reference leg in its correspondent symmetrical configuration. The symmetry group of observability, $G_{C}$, is defined by the symmetrical operations that allows to superimpose the reference leg with the $\kappa$ th leg. In consequence, the symmetry group $G_{C}$ can be derived from the symmetry group of the mechanism $\left(G_{M}\right): G_{C} \subseteq G_{M}$, where

$$
G_{C}=\left\{\lambda_{1}, \lambda_{2}, \ldots, \lambda_{n_{\text {legs }}}\right\} \text {. }
$$

A Jacobian matrix is calculated for each leg to compute the observability, namely:

$$
\begin{array}{r}
\mathbf{C}_{\kappa}^{T}=\left[\begin{array}{lll}
\left(\mathbf{C}_{\kappa}^{1}\right)^{T} \quad\left(\mathbf{C}_{\kappa}^{2}\right)^{T} & \ldots & \left.\left(\mathbf{C}_{\kappa}^{N}\right)^{T}\right]
\end{array} \quad\left(\kappa=1,2, \ldots, n_{\text {legs }}\right),\right. \\
\mathbf{C}_{\kappa}^{j}\left(\varphi_{\kappa}, \mathbf{r}^{j}, \rho^{j}\right)=\frac{\partial F_{\kappa}\left(\varphi_{\kappa}, \mathbf{r}^{j}, \rho^{j}\right)}{\partial \varphi_{\kappa}^{T}} \quad(j=1,2, \ldots, N),
\end{array}
$$

where the $F_{\kappa}$ function is the $\kappa$ th constraint equation of the set of Eqs. $4, \mathrm{r}$ is the mobile platform position, and $\rho$ is a parametric represention of the mobile platform orientation (e.g. a set of Euler angles). Each row of the Jacobian matrix $\mathbf{C}_{\kappa}$ corresponds to a configuration of the mechanism used for KI. Reference [1] presents a $Q R$ decomposition of the Jacobian matrix (Eq. 8) to calculate the observability of the kinematic parameters:

$$
\underline{\mathbf{Q}}^{T} \mathbf{C}_{\kappa}=\left[\frac{\mathbf{R}}{\mathbf{0}}\right]
$$

where $\underline{\mathbf{Q}}$ is a $N \times N$ orthogonal matrix, $\underline{\mathbf{R}}$ is a $n_{\varphi} \times n_{\varphi}$ upper triangular matrix, $\mathbf{0}$ is a $(N-$ $\left.n_{\varphi}\right) \times n_{\varphi}$ zero matrix, and $n_{\varphi}$ is the cardinality of $\varphi_{\kappa}$. The observability of the $i$ th parameter is estimated by its correspondent element on the diagonal of the $\underline{\mathbf{R}}$ matrix. Therefore, the symmetrical observability for a set of $N$ mobile platform poses $\{\mathbf{R}, \mathbf{P}\}$ is stated as:

$$
\begin{array}{r}
\left|\underline{\mathbf{R}}_{i i}^{\kappa}\left(\mathbf{C}_{\kappa}\left(\varphi_{\kappa}, \mathbf{R}, \mathbf{P}\right)\right)\right|=\left|\underline{\mathbf{R}}_{i i}^{1}\left(\mathbf{C}_{1}\left(\varphi_{1}, \lambda_{\kappa}(\mathbf{R}), \mathbf{P}\right)\right)\right| \\
\left(i=1,2, \ldots, n_{\varphi}\right) \quad\left(\kappa=1,2, \ldots, n_{\text {legs }}\right),
\end{array}
$$

where, without loss of generality the first leg is assumed as the reference, $\mathbf{R}$ and $\mathbf{P}$ are column matrices of $N$ position vectors and parametric representations of the orientation of the mobile platform respectively, and $\lambda_{\kappa}$ is the $\kappa$ th symmetry operation of $G_{C}$ that is applied individually over each mobile platform position in $\mathbf{R}$. The parameters with magnitude near to zero are less observable, and the non-observable parameters are those for which $\underline{\mathbf{R}}_{i i}=0$.

The natural use of the symmetrical observability is in KI. A procedure is proposed in section 4.1 to symmetrically design the KI configurations of symmetrical-parallel mechanisms. 


\subsection{Selection of symmetrical configurations for KI}

By the symmetrical planning of the KI of parallel mechanisms it is possible to reduce the optimal posture selection to $1 / n_{\text {legs }}$ of the original searching. Figure 3 presents the procedure.

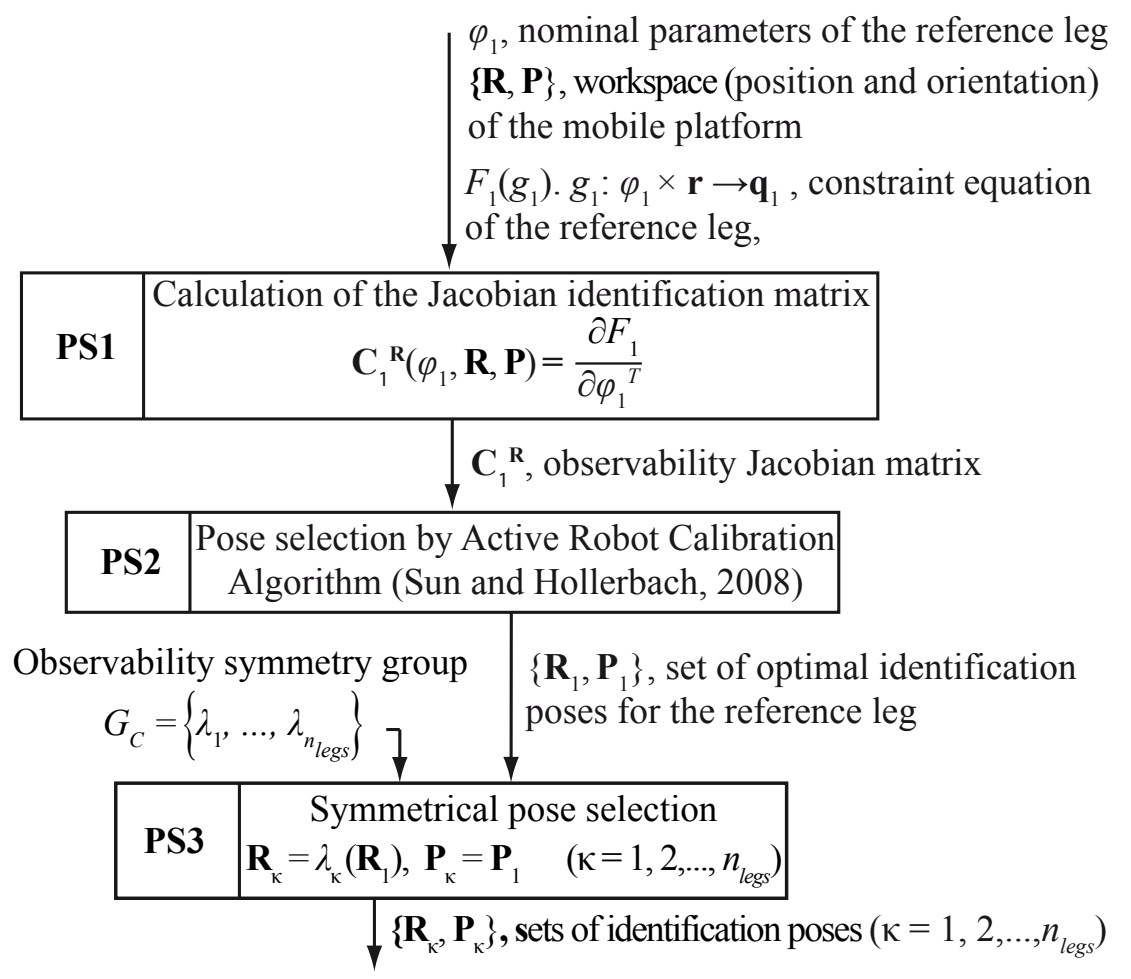

Figure 3. Symmetrical pose selection for KI.

PS1. Calculation of the observability Jacobian matrix. Given: $\varphi_{1}$ the nominal parameters of a reference leg, $F_{1}$ the correspondent constraint kinematic function, and $\{\mathbf{R}, \mathbf{P}\}$ a representative set of workspace poses; To calculate: $\mathbf{C}_{1}^{\mathbf{R}}$ the observability Jacobian matrix,

$$
\mathbf{C}_{1}^{\mathbf{R}}\left(\varphi_{1}, \mathbf{R}, \mathbf{P}\right)=\frac{\partial F_{1}\left(\varphi_{1}, \mathbf{R}, \mathbf{P}\right)}{\partial \varphi_{1}^{T}}
$$

PS2. Given: $\mathbf{C}_{1}^{\mathbf{R}}$ calculated in (PS1.); To select: $\left\{\mathbf{R}_{1}, \mathbf{P}_{1}\right\}$ an optimal set of configurations for the KI of the reference leg. The active calibration algorithm by [11] is adopted to select the configurations. Then, the optimized set of configurations is:

$$
\begin{array}{r}
\max _{\left\{\mathbf{R}_{\mathbf{1}}, \mathbf{P}_{\mathbf{1}}\right\}} O_{1}, \\
\text { subject to : } \mathbf{R}_{1} \subset \mathbf{R}, \mathbf{P}_{1} \subset \mathbf{P},
\end{array}
$$

were $O_{1}$ is an observability index defined by:

$$
O_{1}\left(\mathbf{C}_{1}\left(\varphi_{1}, \mathbf{R}_{1}, \mathbf{P}_{1}\right)\right)=\frac{\left(s_{1} s_{2} \cdots s_{n_{1}}\right)^{1 / n_{\varphi}}}{n_{\varphi}}
$$


$n_{\varphi}$ is the number of parameters to be estimated, and $s_{i}\left(i=1,2, \ldots n_{\varphi}\right)$ are the singular values of the Jacobian matrix. Reference [6] suggest that the number of configurations should be two or three times larger than the number of parameters to be estimated .

PS3. Given: $\{\mathbf{R} 1, \mathbf{P} 1\}$ calculated in (PS2.), and $G_{C}$ the observability symmetry group; To find: the sets of identification configurations of the remaining $\left(n_{\text {legs }}-1\right)$ legs:

$$
\begin{array}{r}
\mathbf{R}_{\kappa}=\lambda_{\kappa}\left(\mathbf{R}_{\mathbf{1}}\right) \quad\left(\kappa=2, \ldots, n_{\text {legs }}\right), \\
\mathbf{P}_{\kappa}=\mathbf{P}_{\mathbf{1}} \quad\left(\kappa=2, \ldots, n_{\text {legs }}\right) .
\end{array}
$$

The KI protocol by [5] is equipped with the symmetrical selection procedure in section 4.2.

\subsection{Symmetrical KI protocol}

Figure 4 presents the main steps of the KI protocol:

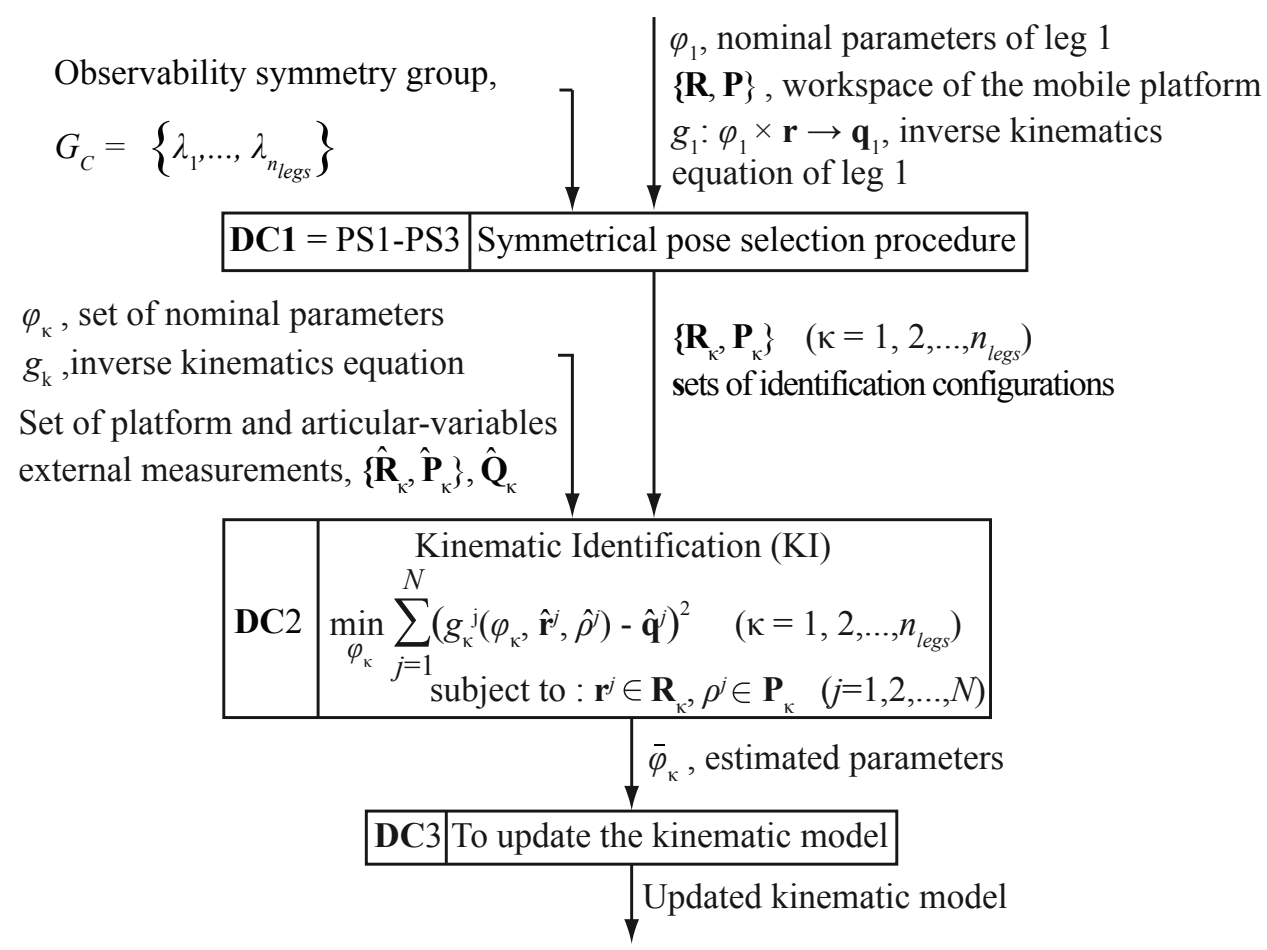

Figure 4. Symmetrical KI protocol for parallel mechanisms.

DC1. Symmetrical pose selection. Given: $\varphi_{\kappa}$ the sets of nominal parameters, $g_{\kappa}$ the inverse kinematic functions, and $\{\mathbf{R}, \mathbf{P}\}$ a representative set of postures of the workspace; To find: $\left\{\mathbf{R}_{\kappa}, \mathbf{P}_{\kappa}\right\}$ the independent sets of configurations that maximizes the observability of $\varphi_{\kappa}\left(\kappa=1,2, \ldots, n_{\text {legs }}\right)$. Compared to [5], a pose selection that takes advantage of the observability symmetries is proposed: the configurations are optimized for a reference leg and the remaining $n_{\text {legs }}-1$ sets are calculated by symmetry operations. The selection procedure is detailed in section 4.1 .

DC2. Kinematic identification. Given: $\left\{\mathbf{R}_{\kappa}, \mathbf{P}_{\kappa}\right\}$ the optimized set of configurations calculated in (DC1.), $\hat{\mathbf{Q}}_{\kappa}=\left[\begin{array}{lll}\hat{q}_{\kappa}^{1} & \cdots & \hat{q}_{\kappa}^{N}\end{array}\right]^{T}$ the set of article-variables measurements, and 
$\left\{\hat{\mathbf{R}}_{\kappa}, \hat{\mathbf{P}}_{\kappa}\right\}$ the set of mobile platform measurements; To solve: the optimization problem defined in Eq. 15 for the estimation of the sets of kinematic parameters $\varphi_{\kappa}(\kappa=$ $\left.1,2, \ldots, n_{\text {legs }}\right)$. The optimization problem is defined in the following manner:

$$
\begin{array}{r}
\min _{\varphi_{\kappa}} \sum_{j=1}^{N}\left(g_{\kappa}^{j}\left(\varphi_{\kappa}, \hat{\mathbf{r}}^{j}, \hat{\rho}^{j}\right)-\hat{q}_{\kappa}^{j}\right)^{2} \quad\left(\kappa=1,2, \ldots, n_{\text {legs }}\right), \\
\text { subject to }: \mathbf{r}^{j} \in \mathbf{R}_{\kappa}, \rho^{j} \in \mathbf{P}_{\kappa} \quad(j=1,2, \ldots, N),
\end{array}
$$

where $\left\{\mathbf{R}_{\kappa} \subset \mathbf{R}, \mathbf{P}_{\kappa} \subset \mathbf{P}\right\} .\{\mathbf{R}, \mathbf{P}\}$ is a workspace constraining the optimization.

DC3. Update of kinematic model. Given the identified sets of parameters obtained in (DC2.) to update the kinematic model of the symmetrical-parallel mechanism.

Section 5 presents the study of workspace, articular-variables space and observability symmetries applied in the KI of a $3 \underline{R} R R$ symmetrical-parallel mechanism.

\section{RESULTS}

Kinematic identification of symmetrical-parallel mechanisms is presented through a $3 \underline{R} R R$ parallel mechanism case study. The mechanism has three degrees of freedom and is illustrated in Fig. 5. It consists on an equilateral moving platform $\left(b_{1} b_{2} b_{3}\right)$, that is connected by three identical revolute - revolute - revolute $(\underline{R} R R)$ kinematic chains $\left(A_{\kappa} C_{\kappa} b_{\kappa}, \kappa=1,2,3\right)$ to an equilateral fixed base $\left(A_{1} A_{2} A_{3}\right)$ as it is presented on Fig. 5). Each kinematic chain is actuated from its fixed joint (articular coordinate). The following set of nominal parameters is assumed:

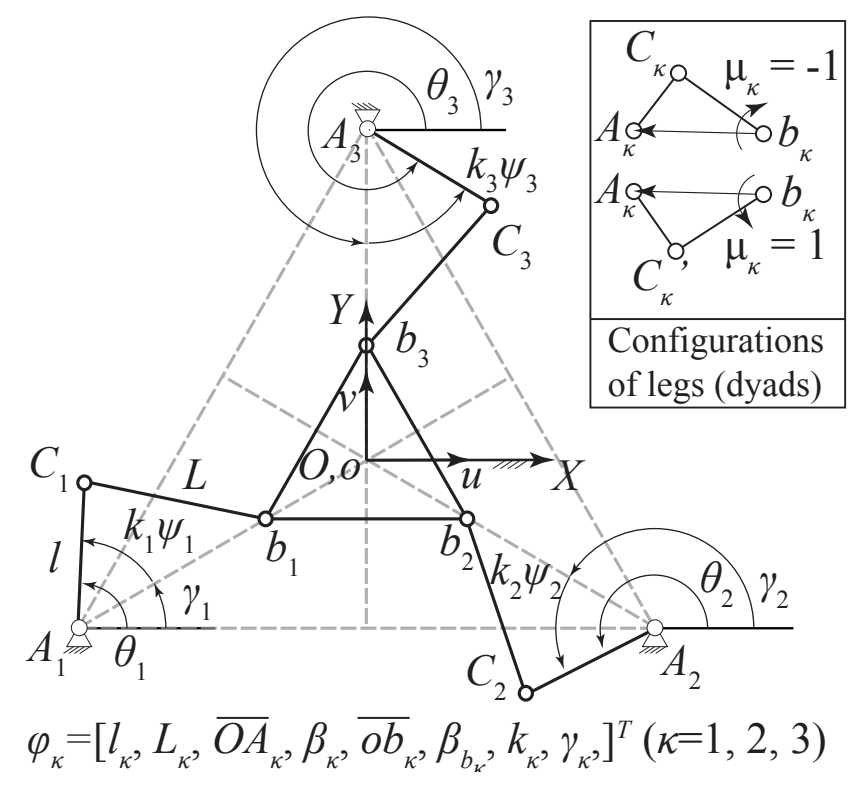

Figure 5. 3RRR symmetrical-parallel mechanism. Symmetrical configuration and kinematic parameters.

1. Dimensions of the links: $A_{1} A_{2}=A_{2} A_{3}=A_{1} A_{3}=6.00 \mathrm{~m}, b_{1} b_{2}=b_{2} b_{3}=b_{1} b_{3}=1.50$ $\mathrm{m}, l=L=1.50 \mathrm{~m}$. 


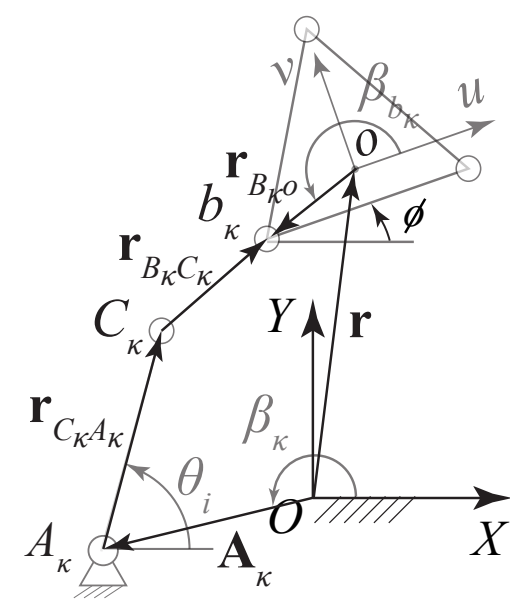

Figure 6. $3 \underline{R} R R$ symmetrical-parallel mechanism. Constraint loop.

2. Configuration of the legs (dyads): $[-1-1-1]$. Each leg is considered as a dyad that can be configured +1 or -1 according to the convention described in Fig. 5 .

3. The nominal gain in the articular coordinate sensors is $k_{1}=k_{2}=k_{3}=1$.

For the kinematic modeling, symmetry analysis and KI, the origin of the fixed coordinate frame is located at the geometric center of the fixed base $A_{1} A_{2} A_{3}$. The $X$-axis points along the direction of $A_{1} A_{2}$ and the $Y$-axis is perpendicular to $A_{1} A_{2}$ (see Fig. 5). A moving frame is attached to the geometric center of the platform. The $u$-axis of the platform frame points along the line $b_{1} b_{2}$, and the $v$-axis is perpendicular to $b_{1} b_{2}$ (see Fig. 5). The location of the moving platform is specified by the coordinates of the platform center and the orientation angle of the moving frame with respect to the fixed frame in the following manner:

$$
\begin{array}{r}
\mathbf{r}=\left[\begin{array}{ll}
x & y
\end{array}\right]^{T}, \\
\rho=\phi .
\end{array}
$$

\subsection{Workspace symmetry}

The $3 \underline{R} R R$ symmetrical-parallel mechanism satisfies the workspace symmetry conditions (1) to (2), section 3:

1. The constraint kinematic equation of each leg is expressed by a closed loop in the following manner, Fig. 6:

$$
\left\|\mathbf{r}-\mathbf{A}_{\kappa}\right\|-\left\|\mathbf{r}_{C_{\kappa} A_{\kappa}}+\mathbf{r}_{B_{\kappa} C_{\kappa}}-\mathbf{r}_{B_{\kappa} o}\right\|=0 \quad(\kappa=1,2,3) .
$$

2. The symmetry group of the mechanism structure $\left(G_{M}\right)$ is determined from Fig. 5:

$$
G_{M}=\left\{\lambda_{1}, \lambda_{2}, \lambda_{3}, \lambda_{4}, \lambda_{5}, \lambda_{6}\right\}
$$

where the first three elements of $G_{M}$ represent rotations about the $Z$-axis: $0 \mathrm{rad}, 2 \pi / 3$ $\mathrm{rad}$, and $4 \pi / 3 \mathrm{rad}$. The last three elements of $G_{M}$ denote reflections about: $O A_{1}, O A_{2}$, and $O A_{3}$ respectively. 
The actuation of the symmetry group $G_{M}$ on the workspace will make it to superimpose with itself. The symmetrical workspace theorem for this mechanism is proved on references $[12,13]$.

\subsection{Articular-variables space symmetry}

An articular-coordinate linear model (Eq.6) with $n_{\text {legs }}=3$ is considered for the $3 \underline{R} R R$ symmetrical-parallel mechanism. In consequence, to design a symmetrical $\mathrm{KI}$ is also necessary to satisfy the condition (3) of articular-variables space symmetry presented in section 3 . Figure 5 shows the symmetrical configuration of the $3 \underline{R} R R$ mechanism that determines the symmetry of the articular-variables space, being the articular-variables vector defined as $\mathbf{q}=\left[\begin{array}{lll}\psi_{1} & \psi_{2} & \psi_{3}\end{array}\right]^{T}$. The mechanism is configured symmetrically positioning the articularcoordinates measuring system in the following manner:

$$
\gamma_{1}=\pi / 6 \mathrm{rad}, \gamma_{2}=5 \pi / 6 \mathrm{rad}, \gamma_{3}=-\pi / 2 \mathrm{rad} .
$$

Figure 7a presents a numerical calculation of the articular-variables space that was performed to verify the symmetry conjecture. Additionally, three constant orientation spaces are evaluated: $\phi=0 \mathrm{rad}, \phi=0.4 \mathrm{rad}, \phi=0.6 \mathrm{rad}$. The results are shown on Figs. $7 \mathrm{~b}$ to $7 \mathrm{~d}$. The symmetry group of the articular-variables space corresponds to rotations of $0 \mathrm{rad}, 2 \pi / 3 \mathrm{rad}$, and $4 \pi / 3 \mathrm{rad}$ around the axis $\psi_{1}=\psi_{2}=\psi_{3}$ (see Fig. 7a)

\subsection{Formulation of symmetrically observable sets of leg parameters}

Nominally, for $(\kappa=1,2,3)$, the set of kinematic parameters is defined by the position of fixed points $\left(\mathbf{A}_{\kappa}\right)$, the position of mobile platform points $\left(\mathbf{b}_{\kappa}\right)$, the leg lengths $\left(l_{\kappa}\right.$ and $\left.L_{\kappa}\right)$, and the articular-coordinate gain and offset $\left(k_{\kappa}\right.$ and $\left.\psi_{\kappa}\right)$. This set of parameters is expressed to be symmetrically observable in consequence with the conditions (1)-(6) of symmetrical observability presented in section 4: The base and platform points are modeled as constrained on the mechanism plane, the fixed base and platform points are defined by the magnitude of the $\overline{O A_{\kappa}}$ and $\overline{o b_{\kappa}}$ segments, and the angles $\beta_{\kappa}$ and $\beta_{b_{\kappa}}$ respectively (see Fig. 6). A linear model (Eq .6) is assumed for the articular variables. In consequence, the set of parameters to be identified is defined in the following manner (see Fig. 5):

$$
\varphi_{\kappa}=\left[l_{\kappa}, L_{\kappa}, \overline{O A_{\kappa}}, \beta_{\kappa}, \overline{o b_{\kappa}}, \beta_{b_{\kappa}}, k_{\kappa}, \gamma_{\kappa}\right]^{T} \quad(\kappa=1,2,3) .
$$

The symmetry observability group, $G_{C} \subseteq G_{M}$, corresponds to the symmetry operations that allows the leg 1 to superimpose with the $\kappa$ th leg $(\kappa=1,2,3)$ :

$$
G_{C}=\left\{\lambda_{1}, \lambda_{2}, \lambda_{3}\right\} \quad\left(G_{C} \subseteq G_{M}\right),
$$

where $G_{M}$ is the symmetry group of the mechanism defined by the Eq. 18 .

\subsection{Symmetrical pose selection for kinematic identification}

The mechanism is symmetric as is probed in sections 5.1 and 5.2, and the sets of leg parameters were declared in order to obtain a symmetrical observability. The correspondent symmetry observability group is defined by Eqs. 20-21. Prior to perform the KI, the symmetrical pose selection procedure (section 4.1) is applied: 


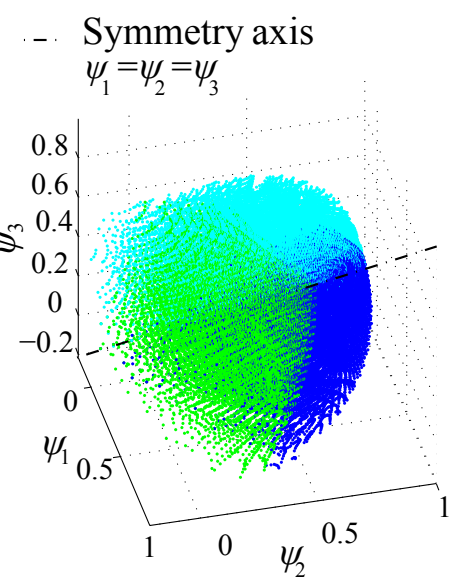

a. Articular-coordinates space obtained by symmetricaloperations around axis

$$
\psi_{1}=\psi_{2}=\psi_{3}
$$

Articular-coordinates space obtained by symmetrical operations

$\diamond \quad$ Maximal error $=9.4937 \cdot 10^{-015} \quad$ Articular-coord space error [rad]
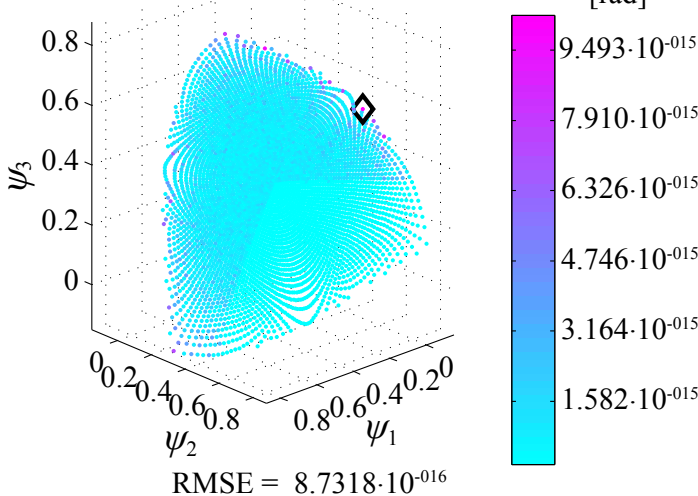

c. Articular-coordinates space associated to end-effector constant orientation $\phi=0.4 \mathrm{rad}$
Articular-coordinates space obtained

by symmetrical operations

$\diamond \quad$ Maximal error $=1.1214 \cdot 10^{-014}$ Articular-coord

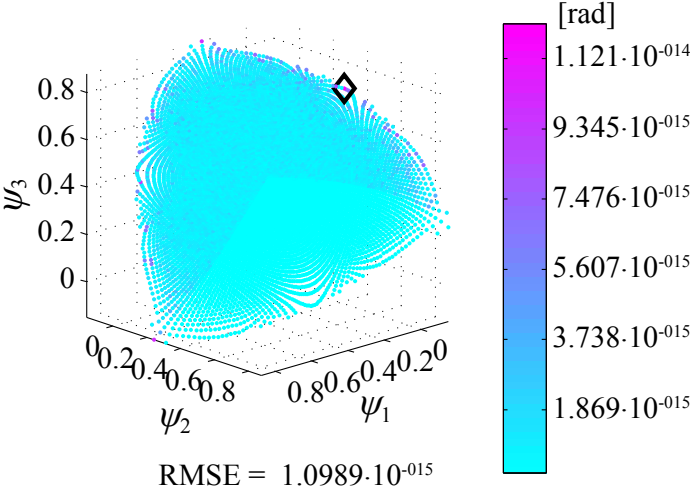

b. Articular-coordinates space associated to end-effector constant orientation $\phi=0 \mathrm{rad}$

Articular-coordinates space obtained by symmetrical operations

$\diamond \quad$ Maximal error $=1.2669 \cdot 10^{-014}$ Articular-coord . space error

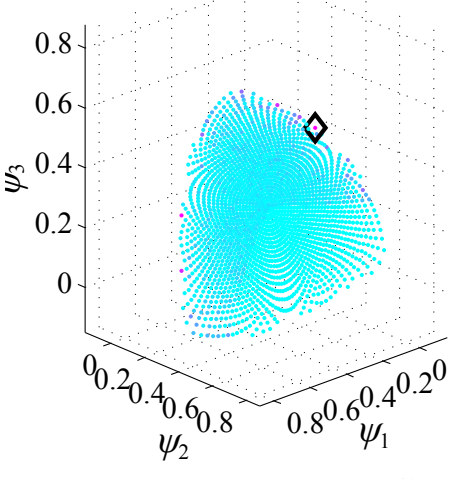
$[\mathrm{rad}]$

$1.267 \cdot 10^{-014}$ $1.055 \cdot 10^{-014}$ $8.446 \cdot 10^{-015}$ $6.335 \cdot 10^{-015}$ $4.223 \cdot 10^{-015}$ $2.111 \cdot 10^{-015}$

d. Articular-coordinates space associated to end-effector constant orientation $\phi=0.6 \mathrm{rad}$

Kinematic parameters: $\bar{A}_{1} A_{2}={\overline{A_{2} A}}_{3}={\overline{A_{1} A_{3}}}_{3}=6.00 \mathrm{~m},{\overline{b_{1} b_{2}}}_{2}={\overline{b_{2} b_{3}}}_{=b_{1} b_{3}}=1.50 \mathrm{~m}$ $l=L=1.50 \mathrm{~m}, k_{1}=k_{2}=k_{3}=1.00 \mathrm{~m}$. Dyads configuration $=\left[\begin{array}{lll}-1 & -1 & -1\end{array}\right]$

Figure 7. $3 \underline{R} R R$ symmetrical-parallel mechanism. Articular-coordinates space obtained by symmetrical operations.

PS1. Calculation of the identification matrix $\left(\mathbf{C}_{1}^{\mathbf{R}}\left(\varphi_{1}, \mathbf{R}, \mathbf{P}\right)\right.$ - Eq. 11). The nominal set of parameters $\left(\varphi_{1}\right)$ is given by the set of conditions (1) - (3) presented in section 5 . The inverse kinematic function $\left(g_{1}\right)$ is given by the Eq. 17 with $\kappa=1$. The workspace $(\{\mathbf{R}, \mathbf{P}\})$ was approximated by a set of 30000 singularity-free poses.

PS2. Selection of optimal KI configurations. A set of 24 optimal KI configurations $\left(\left\{\mathbf{R}_{1}, \mathbf{P}_{1}\right\}\left(\mathbf{C}_{1}^{\mathbf{R}}\right)\right)$ was selected using the active calibration algorithm by [11]. The optimized identification poses are registered in Fig. 8.

PS3. Symmetrical pose selection. The optimal sets of KI configurations for the second and 
third legs are obtained by symmetrical operations over the set $\left\{\mathbf{R}_{1}, \mathbf{P}_{1}\right\}$ :

$$
\begin{array}{r}
\mathbf{R}_{\kappa}=\lambda_{\kappa}\left(\mathbf{R}_{1}\right) \\
\mathbf{P}_{\kappa}=\mathbf{P}_{1} \quad(\kappa=2,3), \\
\end{array}
$$

where the symmetry operations, $\lambda_{\kappa}$ are defined by the Eq. 21 . The symmetrical observability of the legs is verified by the calculation of the observability index (Eq. 10) in Fig. 9.

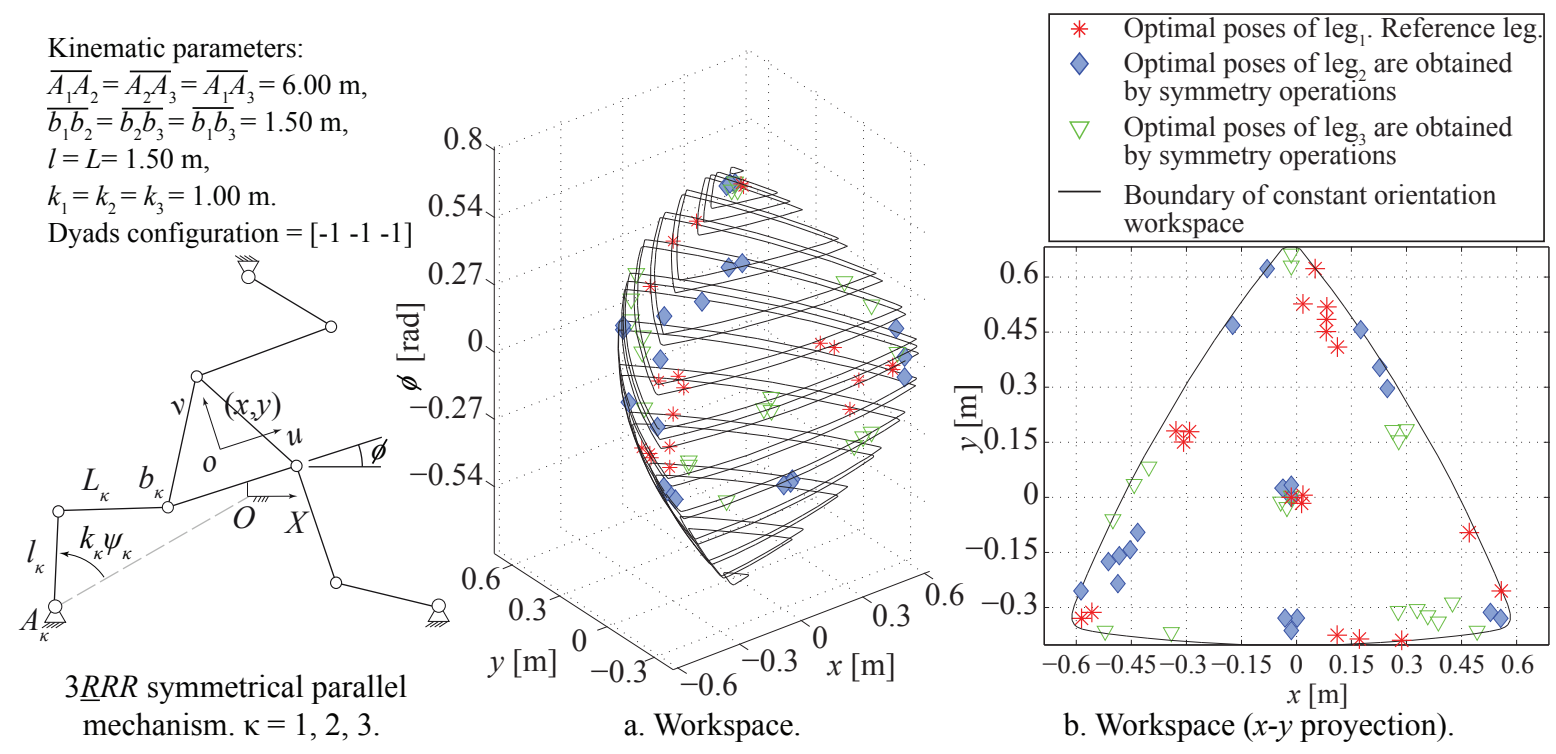

Figure 8. $3 \underline{R} R R$ symmetrical-parallel mechanism. Selected poses for kinematic identification.

Kinematic parameters: ${\overline{A_{1} A_{2}}}_{2}={\overline{A_{2} A_{3}}}_{=\bar{A}_{1} A_{3}}=6.00 \mathrm{~m},{\overline{b_{1} b_{2}}}_{2}{\overline{b_{2}}}_{3}={\overline{b_{1} b}}_{3}=1.50 \mathrm{~m}$ $l=L=1.50 \mathrm{~m}, k_{1}=k_{2}=k_{3}=1.00 \mathrm{~m}$. Dyads configuration $=\left[\begin{array}{lll}-1 & -1 & -1\end{array}\right]$

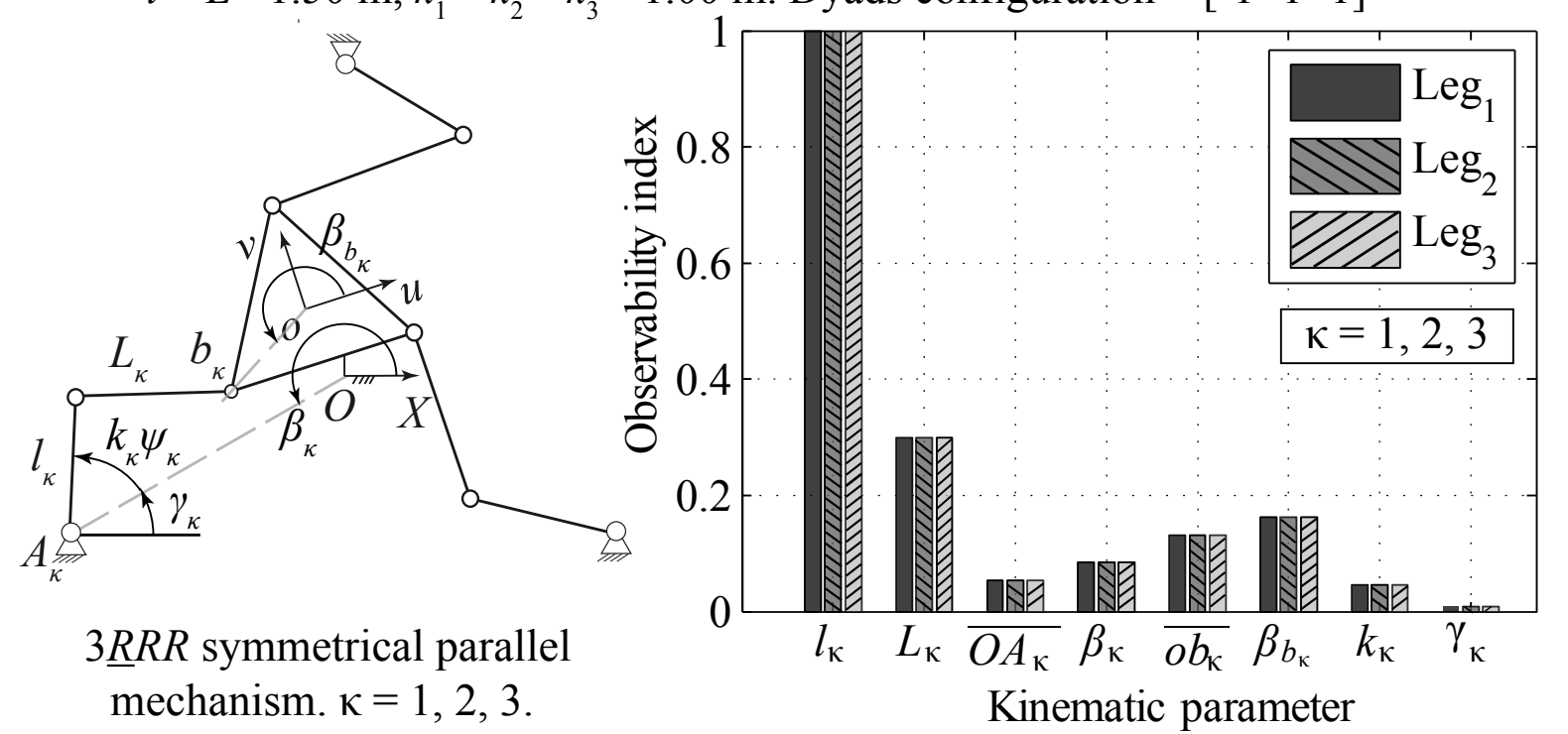

Figure 9. $3 \underline{R} R R$ symmetrical-parallel mechanism. Observability of kinematic parameters.

The kinematic identification is carried out once the identification poses are selected. 


\subsection{Kinematic identification}

The kinematic calibration is simulated to evaluate the performance of the improved KI protocol. The nominal kinematic parameters of the mechanism are disturbed adding random errors with normal distribution and standard deviation $\sigma$ in order to simulate the kinematic errors to be identified. The mobile platform measurements $\left(\hat{\mathbf{R}}_{\kappa}\right)$ are simulated from its correspondent articular-coordinate measurements $\left(\hat{\mathbf{Q}}_{\kappa}\right)$ through a forward kinematics model added with normally distributed random disturbances. The standard deviations of the measurements were defined in the following manner:

$$
\begin{gathered}
\sigma_{r}=1.00 \cdot 10^{-4} \mathrm{~m}, \\
\sigma_{\rho}=1.00 \cdot 10^{-4} \mathrm{rad},
\end{gathered}
$$

where $\sigma_{r}$ and $\sigma_{\rho}$ are the standard deviations in position and orientation measurements respectively. The identification procedure is summarized as:

DC1. Symmetrical pose selection. The symmetrical pose selection is detailed in section 5.4.

DC2. Kinematic parameters identification. A linearization of the inverse kinematics is used to solve the non-linear optimization problem of each leg (Eq. 15):

$$
\Delta \mathbf{Q}_{\kappa}=\mathbf{C}_{\kappa}\left(\varphi_{\kappa}, \mathbf{R}_{\kappa}, \mathbf{P}_{\kappa}\right) \Delta \varphi_{\kappa} \quad(\kappa=1,2,3),
$$

where $\Delta \mathbf{Q}_{\kappa}=\mathbf{Q}_{\kappa}\left(\varphi_{\kappa}, \hat{\mathbf{R}}_{\kappa}, \hat{\mathbf{P}}_{\kappa}\right)-\hat{\mathbf{Q}}_{\kappa}$ is the error in the articular-coordinate and $\Delta \varphi_{\kappa}$ is the set of parameters to be estimated. The estimation is achieved using a iterative linear least-squares solution of Eq. 24:

$$
\Delta \varphi_{\kappa}=\left(\mathbf{C}_{\kappa}^{T} \mathbf{C}_{\kappa}\right)^{-1} \mathbf{C}_{\kappa}^{T} \Delta \mathbf{Q}_{\kappa} \quad(\kappa=1,2,3) .
$$

DC3. Update the kinematic model with the set of estimated parameters $\bar{\varphi}=\left\{\bar{\varphi}_{1}, \bar{\varphi}_{2}, \bar{\varphi}_{3}\right\}$ where

$$
\bar{\varphi}_{\kappa}=\varphi_{\kappa}+\Delta \varphi_{\kappa} \quad(\kappa=1,2,3) .
$$

The performance of the identification is evaluated after the kinematic calibration by means of the calculation the root mean square (RMSE) of the difference between the commanded mobile platform pose $(\{\mathbf{R}, \mathbf{P}\})$ and a correspondent set of simulated measurements $(\{\hat{\mathbf{R}}, \hat{\mathbf{P}}\})$. The set of measured configurations corresponds to the set of 30000 configurations used to approximate the workspace and articular-variables space. An alternative traditional inverse kinematic calibration is performed using 24 poses optimized for the identification of the complete set of parameters of the mechanism (optimal for all the legs). The set of optimal configurations is selected using the same active robot calibration algorithm as in the case of the symmetrical KI protocol. The results are registered in: Figure 10 presents the RMSE of the mobile platform pose estimated from the workspace before and after calibration, and Figure 11 presents the local platform position errors calculated for constant orientation spaces $(\phi=0.0 \mathrm{rad}, \phi=0.4 \mathrm{rad}, \phi=0.6 \mathrm{rad})$.

Concluding remarks of the use of observability symmetries in kinematic identification of parallel mechanisms are presented in section 6 . 


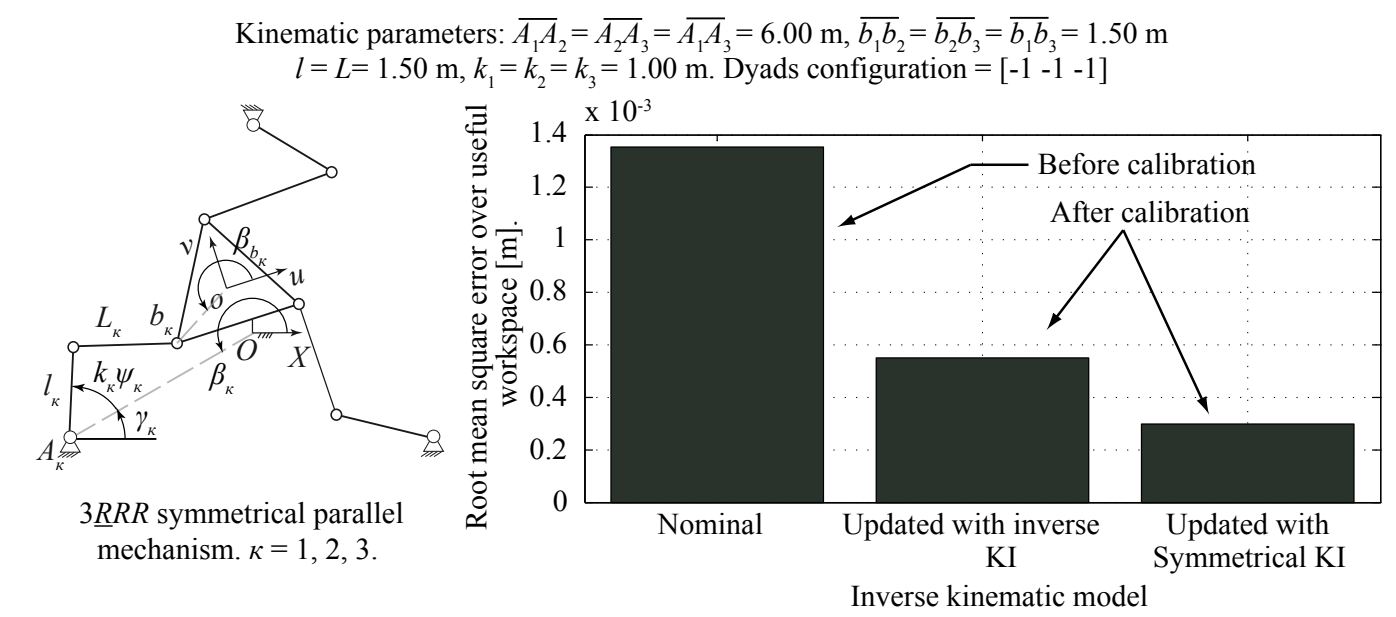

Figure 10. $3 \underline{R} R R$ symmetrical-parallel mechanism. Estimated mobile platform pose rootmean-square error for a singularity-free workspace.

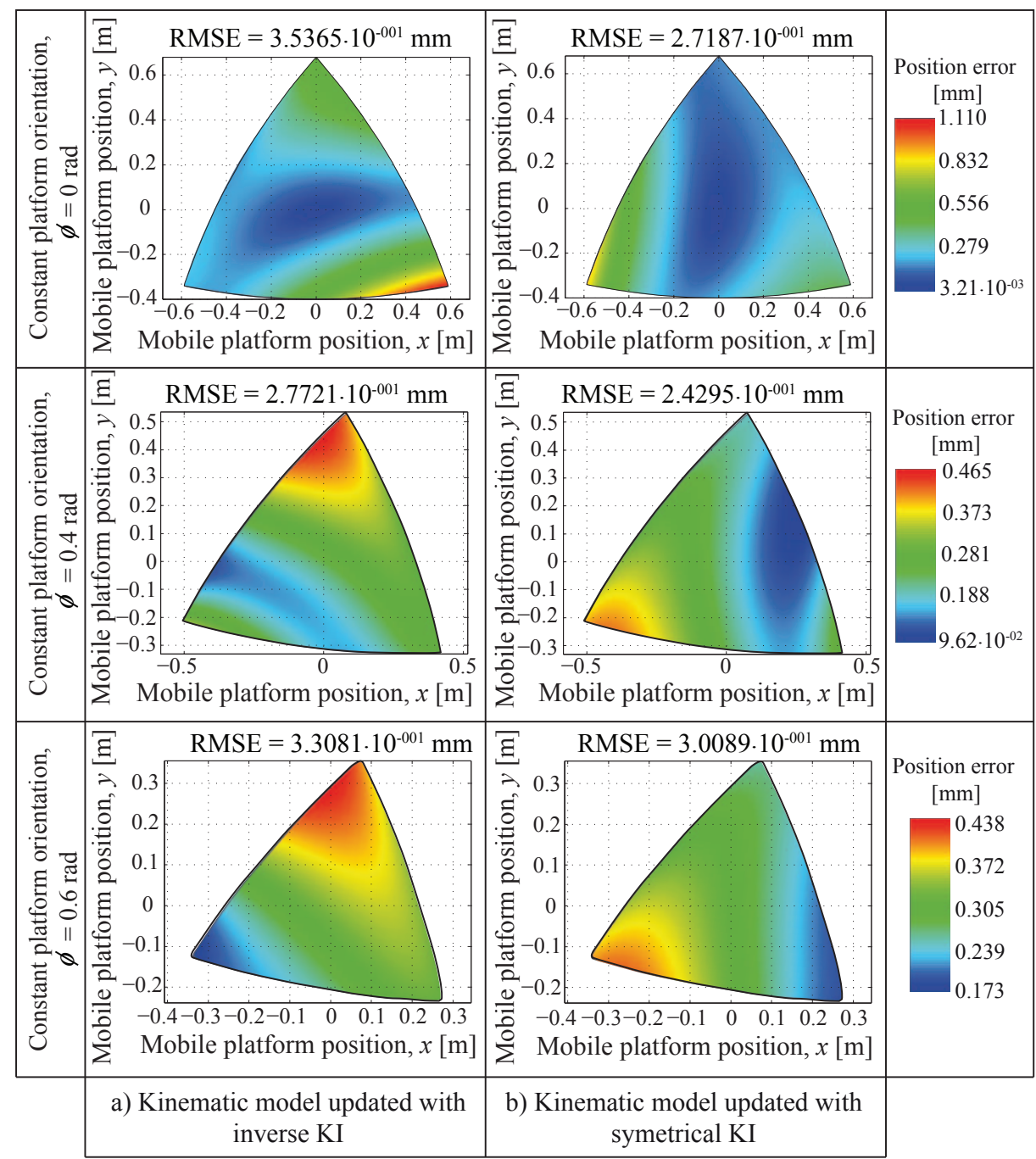

Kinematic parameters: ${\overline{A_{1} A}}_{2}={\overline{A_{2} A}}_{3}={\overline{A_{1} A_{3}}}_{3}=6.00 \mathrm{~m},{\overline{b_{1} b_{2}}}_{2}={\overline{b_{2}}}_{3}={\overline{b_{1} b_{3}}}_{3}=1.50 \mathrm{~m}$

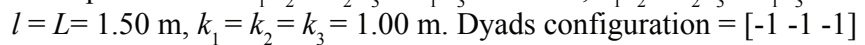

Figure 11. 3RRR symmetrical-parallel mechanism. Estimated mobile platform position error on constant orientation workspaces. 


\section{CONCLUSION}

This article addresses the problem of declaring sets of leg parameters with symmetrical observability for symmetrical-parallel mechanisms. The necessary conditions for the symmetrical observability are proposed in section 4 and summarized in the following manner:

1. The mechanism has a symmetric structure and symmetrical workspace characterized by the symmetry groups $G_{M}$ and $G_{W}$ respectively (section 4 - (1)).

2. The kinematic joints are modeled as perfectly assembled and in the case of planar mechanisms the links are assumed to be constrained in the mechanism plane (section 4 - (2)).

3. The base and platform joint parameters of each leg are defined in order to obtain a symmetrical observability with respect to the workspace (section 4 - (3), (4)).

4. If a linear model (Eq. 6) is assumed for the articular coordinates, then additional conditions are required: each articular coordinate has the same nominal gain andthe mechanism has configured a symmetrical articular-variables space $G_{Q}$ (section 4 - (5 - 6)).

To prove the articular-coordinates space symmetry results in a problem analogous to the forward kinematics of parallel mechanisms: it requires the solution of the constraint kinematic equations given the vector of articular variables (see Eq. 5). In general it is not possible to formulate an analytical solution of the forward kinematics of parallel mechanisms (see [8]). In consequence, the mapping of the structural symmetry to the articular-coordinates space symmetry of symmetrical-parallel mechanisms is established as a conjecture (see section 3.1).

A natural use for the symmetrical observability would be the KI in which the experiments are designed for a reference leg only and extended to the remaining legs by symmetrical operations. The KI protocol by [5] was equipped with a selection of symmetrical configurations procedure based on the formulation of symmetrically observable sets of leg parameters. Compared with [5], the symmetrical pose selection allows to reduce the design of experiment costs to $1 / n_{\text {legs }}$. The procedure is developed in section 4.1 and summarized in the following manner:

PS1. Calculation of an observability Jacobian matrix over the workspace.

PS2. Selection of set of optimal identification poses of a reference leg. The pose selection is calculated using the active calibration algorithm by [11].

PS3. Determination of the optimal configurations for the remaining $n_{\text {legs }}-1$ by the symmetrical observability operations over the reference set.

The KI protocol is presented in section 4.2. Compared to traditional identification strategies the protocol has the following advantages:

1. The cost reduction in the design of identification experiments by the use of observability symmetries. 
2. The improvement of the numerical efficiency of the procedure for the selection of optimal KI configurations by the adoption of the active robot calibration algorithm by [11].

3. As a consequence of (1) - (2), the improvement of the kinematic identification results.

Discussion. This article presents a KI protocol based on the structural symmetries of parallel mechanisms. However, parallel mechanisms are imperfectly symmetrical in practice. This is not a concern for KI: even if the mechanism is imperfectly symmetric, the observability Jacobian matrix of each leg will be calculated with the set of nominal (perfect) parameters as in the case of a regular inverse KI. This is based on the negligible change of the nominal Jacobian with respect to the actual one.

Future work. The KI of symmetrical-parallel mechanism should be probed on a experimental set-up.

\section{Acknowledgements}

The authors wish to acknowledge the financial support for this research by the Colombian Administrative Department of Sciences, Technology and Innovation (COLCIENCIAS), and the Colombian National Service of Learning (SENA), grant 1216-479-22001.

\section{REFERENCES}

[1] S. Besnard and W. Khalil. Identifiable parameters for parallel robots kinematic calibration. In IEEE International Conference on Robotics and Automation - ICRA, volume 3, 2001.

[2] I.A. Bonev and C.M. Gosselin. Analytical determination of the workspace of symmetrical spherical parallel mechanisms. IEEE Trans. Rob., 22(5):1011 -1017, oct. 2006.

[3] D. Daney. Optimal measurement configurations for Gough platform calibration. In International Conference on Robotics and Automation ICRA'02, volume 1, pages 147152. IEEE, 2002.

[4] D. Daney. Kinematic calibration of the Gough platform. Robotica, 21(06):677-690, 2003.

[5] S. Durango, D. Restrepo, O. Ruiz, J. Restrepo-Giraldo, and S. Achiche. Kinematic identification of parallel mechanisms by a divide-and-conquer strategy. In 7th International Conference on Informatics in Control, Automation and Robotics - ICINCO, volume 2, pages $167-173,2010$.

[6] J.H. Jang, S.H. Kim, and Y.K. Kwak. Calibration of geometric and non-geometric errors of an industrial robot. Robotica, 19(03):311-321, 2001.

[7] J.P. Merlet. Parallel robots, chapter 1, pg. 13. Springer-Verlag New York Inc, 2006. 
[8] P. Renaud, A. Vivas, N. Andreff, P. Poignet, P. Martinet, F. Pierrot, and O. Company. Kinematic and dynamic identification of parallel mechanisms. Control Eng. Prac., 14(9):1099-1109, 2006.

[9] O. Ruiz and C. Cadavid. Geometrics functions in computer aided geometric design. Fondo Editorial Universidad EAFIT, first edition, 2008.

[10] B. Siciliano and O. Khatib, editors. Springer Handbook of Robotics. Springer, Berlin, 2008.

[11] Y. Sun and J.M. Hollerbach. Active robot calibration algorithm. In International Conference on Robotics and Automation - ICRA, pages 1276-1281, May 2008.

[12] J.-S. Zhao, M. Chen, K. Zhou, J.-X. Dong, and Z.-J. Feng. Workspace of parallel manipulators with symmetric identical kinematic chains. Mech. Mach. Theory, 41(6):632 $-645,2006$.

[13] J.-S. Zhao, F. Chu, and Z.-J Feng. Symmetrical characteristics of the workspace for spatial parallel mechanisms with symmetric structure. Mech. Mach. Theory, 43(4):427 $-444,2008$.

[14] J.-S. Zhao, F. Chu, and Z-J Feng. Singularities within the workspace of spatial parallel mechanisms with symmetric structures. P. I. Mech. Eng. C-J. Mec., 224(2):459-472, 2010. 